Tietjen, Sabrina; Thünemann, Silvia

\title{
Forschungswerkstatt digital. Ein modernes Lehr-Lernarrangement für eine Digitalisierungsstrategie im Lehramt?
}

Holub, Barbara [Hrsg.]; Himpsl-Gutermann, Klaus [Hrsg.]; Mittlböck, Katharina [Hrsg.]; Musilek-Hofer, Monika [Hrsg.]; Varelija-Gerber, Andrea [Hrsg.]; Grünberger, Nina [Hrsg.]: lern.medien.werk.statt. Hochschullernwerkstätten in der Digitalität. Bad Heilbrunn : Verlag Julius Klinkhardt 2021, S. 147-159. - (Lernen und Studieren in Lernwerkstätten)

Quellenangabe/ Reference:

Tietjen, Sabrina; Thünemann, Silvia: Forschungswerkstatt digital. Ein modernes Lehr-Lernarrangement für eine Digitalisierungsstrategie im Lehramt? - In: Holub, Barbara [Hrsg.]; Himpsl-Gutermann, Klaus [Hrsg.]; Mittlböck, Katharina [Hrsg.]; Musilek-Hofer, Monika [Hrsg.]; Varelija-Gerber, Andrea [Hrsg.]; Grünberger, Nina [Hrsg.]: lern.medien.werk.statt. Hochschullernwerkstätten in der Digitalität. Bad Heilbrunn : Verlag Julius Klinkhardt 2021, S. 147-159 - URN: urn:nbn:de:0111-pedocs-228189 - DOI: 10.25656/01:22818

https://nbn-resolving.org/urn:nbn:de:0111-pedocs-228189

https://doi.org/10.25656/01:22818

in Kooperation mit / in cooperation with:

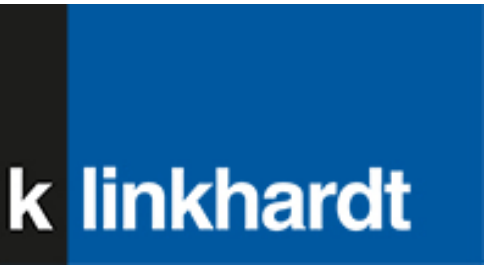

http://www.klinkhardt.de

\section{Nutzungsbedingungen}

Dieses Dokument steht unter folgender Creative Commons-Lizenz: http://creativecommons.org/licenses/by-nc-sa/4.0/deed.de - Sie dürfen das Werk bzw. den Inhalt unter folgenden Bedingungen vervielfältigen, verbreiten und öffentlich zugänglich machen sowie Abwandlungen und Bearbeitungen des Werkes bzw. Inhaltes anfertigen: Sie müssen den Namen des Autors/Rechteinhabers in der von ihm festgelegten Weise nennen. Dieses Werk bzw, der Inhalt darf nicht für kommerzielle Zwecke verwendet werden. Die neu entstandenen Werke bzw. Inhalte dürfen nur unter Verwendung von Lizenzbedingungen weitergegeben werden, die mit denen dieses Lizenzbedingungen weitergegeben werden,

Mit der Verwendung dieses Dokuments erkennen Sie die Nutzungsbedingungen an.

\section{Terms of use}

This document is published under following Creative Commons-License: http://creativecommons.org/licenses/by-nc-sa/4.0/deed.en - You may copy, distribute and transmit, adapt or exhibit the work in the public and alter, transform or change this work as long as you attribute the work in the manner specified by the author or licensor. You are not allowed to make commercial use of the work. If you alter, transform, or change this work in any way, you may distribute the resulting work only under this or a comparable license.

By using this particular document, you accept the above-stated conditions of

\section{Kontakt / Contact:}

\section{peDOCS}

DIPF | Leibniz-Institut für Bildungsforschung und Bildungsinformation Informationszentrum (IZ) Bildung

E-Mail: pedocs@dipf.de

Internet: www.pedocs.de

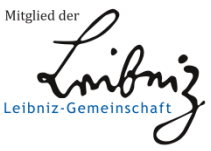




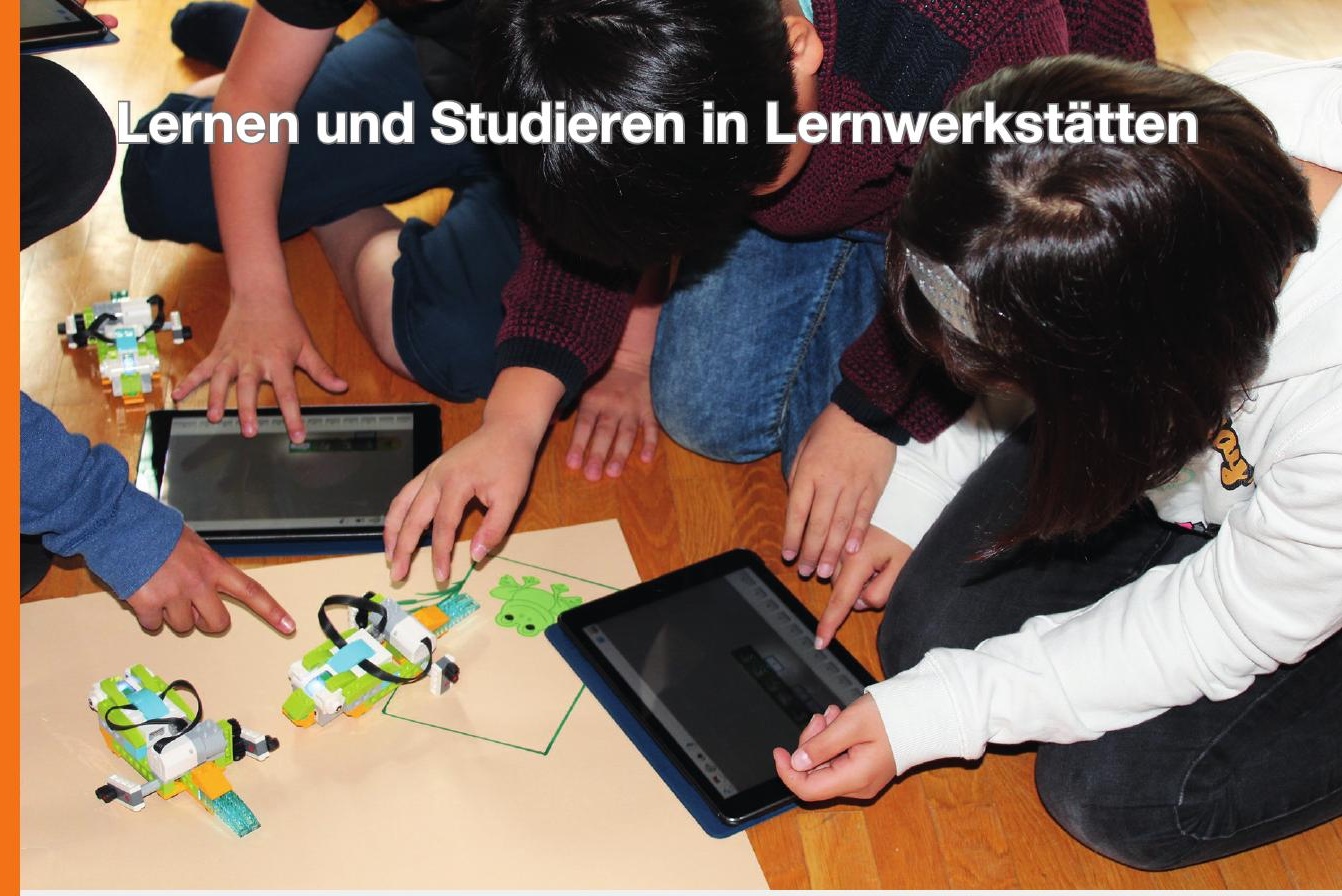

Barbara Holub / Klaus Himpsl-Gutermann Katharina Mittlböck / Monika Musilek-Hofer Andrea Varelija-Gerber / Nina Grünberger (Hrsg.)

\section{lern.medien.werk.statt}

Hochschullernwerkstätten in der Digitalität 
Barbara Holub Klaus Himpsl-Gutermann Katharina Mittlböck Monika Musilek-Hofer Andrea Varelija-Gerber

Nina Grünberger (Hrsg.)

\section{lern.medien.werk.statt}

Hochschullernwerkstätten in der Digitalität 
Dieser Titel wurde in das Programm des Verlages mittels eines Peer-Review-Verfahrens aufgenommen. Für weitere Informationen siehe www.klinkhardt.de.

Bibliografische Information der Deutschen Nationalbibliothek Die Deutsche Nationalbibliothek verzeichnet diese Publikation in der Deutschen Nationalbibliografie; detaillierte bibliografische Daten sind im Internet abrufbar über http://dnb.d-nb.de.

2021.ig. (C) by Julius Klinkhardt.

Coverfoto: (c) ZLI PH Wien.

Druck und Bindung: AZ Druck und Datentechnik, Kempten.

Printed in Germany 2021.

Gedruckt auf chlorfrei gebleichtem alterungsbeständigem Papier.

Die Publikation (mit Ausnahme aller Fotos, Grafiken und Abbildungen) ist veröffentlicht unter der Creative Commons-Lizenz: CC BY-NC-SA 4.0 International https://creativecommons.org/licenses/by-nc-sa/4.0/

ISBN 978-3-7815-5904-2 digital doi.org/10.35468/5904

ISBN 978-3-7815-2468-2 print 


\section{Inhaltsverzeichnis}

Barbara Holub, Klaus Himpsl-Gutermann, Katharina Mittlböck, Monika Musilek-Hofer, Andrea Varelija-Gerber und Nina Grünberger

Einführung in den Band 11

\section{Zur Konstituierung von Hochschullernwerkstätten}

Sandra Tänzer

Mut - Zumutung - Ermutigung. Oder wie man lernt, eine Hochschullernwerkstatt zu lieben

Markus Peschel, Hartmut Wedekind, Pascal Kihm und Mareike Kelkel

Hochschullernwerkstätten und Lernwerkstätten -

Verortung in didaktischen Diskursen

Dietlinde Rumpf und Corinna Schmude

Von der Herausforderung, die Vielfalt von Hochschullernwerkstätten

in einer Definition abzubilden

Pascal Kihm und Markus Peschel

„Komplexität wagen!“ - Methoden zur Beforschung von

offenen Lehr-Lern-Prozessen in Hochschullernwerkstätten 70

\section{Zum Spannungsverhältnis von Hochschullernwerkstätten und Digitalität}

Mark Weißhaupt, Ralf Schneider, Clemens Griesel und Agnes Pfrang

Digitale Erfahrung? Über das Lernen zwischen Instruktion und

(Ko-)Konstruktion

Stefan Brée, Lena S. Kaiser und Tanja Wittenberg

Lernwerkstatt als digitaler Erfahrungsort. Potenziale und

Herausforderungen für Lernwerkstätten als Orte

„offener multimedialer Produktionsästhetik“

\section{Ulrike Stadler-Altmann und Gerda Winkler}

Real \& virtuell, analog \& digital: Dimensionen einer Kooperation.

Multifunktionalität als Kennzeichen zukunftsfähiger Lernwerkstatt- und

Bibliothekskonzeption 


\section{Josef Buchner und Michael Kerres}

Lernwerkstattarbeit in der digital vernetzten Welt.

Die Perspektive der gestaltungsorientierten Mediendidaktik

Sabrina Tietjen und Silvia Thünemann

Forschungswerkstatt digital: ein modernes Lehr-Lernarrangement

für eine Digitalisierungsstrategie im Lehramt?

\section{Zu fach- und mediendidaktischen Perspektiven}

Johannes Mayer, Antonia Lemensieck, Maria Reinhardt und Karl Wollmann

Fachliche Perspektiven auf digitalisierungsbezogene Lernangebote

in der Ausbildung von Grundschullehrer*innen

Heike Hagelgans

Die Thematisierung digitaler Medien in den schulpraktischen Studien

Fachdidaktische Reflexionen von digitalen Medien für das Lernen im

Mathematikunterricht der Primarstufe

Jeanette Hoffmann, Katharina Egerer und Franziska Herrmann

Analoge Bilder - digitaler Film.

Möglichkeitsräume für literarästhetisches Lernen in

Hochschullernwerkstätten

Michael Rieseneder und Wolfgang Wagner

Erstes Programmieren mit Kindern über Handlungserfahrungen.

Das Konzept Activity-based-Coding

Patrick Isele und Julia Höke

Reflexionen über digitales Lernen mit dem Sphero SPRK+

Erfahrungen mit Studierenden in der „Lernwerkstatt ${ }^{3 “}$

Susanne Schumacher, Ulrike Stadler-Altmann und Enrico A. Emili

Piktogramme als Unterstützungsmedien.

Studien zu Effekten von Bilderbüchern mit zusätzlichen Symbolen im Kindergarten 


\section{Zu Aspekten, Perspektiven und Herausforderungen von Hochschullernwerkstätten}

Sabine Fischer und Max de Baey-Ernsten

Didaktische Perspektiven einer ästhetischen Werkstatt

Tanja Wittenberg und Lena S. Kaiser

„Ich war frustriert, ich bin kein Kind mehr“ - Erfahrungslernprozesse

mit verwendungs- und bedeutungsoffenem Material in

kindheitspädagogischen Hochschullernwerkstätten

Lisa Eßel und Laura Schlichting

Hochschullernwerkstätten im Spannungsfeld zwischen

Freiwilligkeit und Pflicht

Mareike Kelkel, Markus Peschel und Pascal Kihm

Potenziale der pädagogisch-didaktischen Öffnung in

Hochschullernwerkstätten

Barbara Holub und Sybille Roszner

Hochschullernwerkstatt - Ausgangspunkt für

persönlichkeitsorientierte Professionalisierung in der Ausbildung

für Lehrpersonen

Verzeichnis der Autor*innen 
Sabrina Tietjen und Silvia Thünemann

\title{
Forschungswerkstatt digital: ein modernes Lehr-Lernarrangement für eine Digitalisierungsstrategie im Lehramt?
}

\begin{abstract}
Die bremische Forschungswerkstatt Erziehungswissenschaft (ForwerkEW) versteht sich als Ort des Forschenden Studierens in den lehramtsbezogenen Studiengängen an der Universität Bremen. Im Kontext einer Digitalisierung in der Lehrer*innenbildung stellt der Aufsatz die Frage, wie der Werkstattgedanke mit digitalen Elementen verstärkt werden kann und welche Chancen bzw. Risiken damit verbunden sind. Der Beitrag zeigt auf, wie die ForwerkEW mit einer Online-Plattform zum Forschenden Studieren versucht, auf folgende drei aktuelle Herausforderungen der Lehrer*innenbildung zu reagieren: die gebotene Ausweitung von Forschungsbezügen in der Lehre (2.1), die Förderung digitaler Medienkompetenzen (2.2) sowie angesichts der Heterogenität Studierender die Entwicklung inklusiver Lehr-Lernformate (2.3). Nach einer kurzen theoretischen Einführung zu den drei Herausforderungsbereichen wird in Kapitel 3 die bremische Online-Plattform BOOC (Blended Open Online Courses) vorgestellt. BOOC unternimmt den Versuch, die genannten Herausforderungen in einem OnlineStudienangebot zum Forschenden Studieren zusammenzudenken. In einem letzten Schritt hinterfragen wir kritisch die Verknüpfung der Konzepte Forschendes Studieren, Digitalisierung und inklusive Lehr-Lernformate hinsichtlich ihrer Potenziale, aber auch hinsichtlich ihrer Grenzen und möglicher Überforderungsstrukturen.
\end{abstract}

\section{Einleitung: Die Forschungswerkstatt EW und ihr Verständnis vom Forschenden Studieren in der reflexiven Lehrer*innenbildung}

Forschungswerkstätten sind „enge Verwandte“ von Lernwerkstätten. Sie können unter der Prämisse des Forschens auch in modularisierten Studiengängen besondere Lern- und Erprobungsräume sein, wenn sie sich nicht nur als Interpretationssetting verstehen, sondern in ihren Konzeptionen klassische Prinzipien 
von Lernwerkstätten wie Entdecken, Handeln, Reflektieren, Autonomie und Kooperation (Müller-Naendrup, 1997) zu finden sind. Die Forschungswerkstatt Erziehungswissenschaft (ForwerkEW) an der Universität Bremen verpflichtet sich diesem Selbstverständnis von Lernwerkstätten und versteht sich zugleich als Ort und Konzept des Forschenden Studierens (siehe 2.1) in der reflexiven Lehrer*innenbildung der Universität Bremen. Als Ort steht sie im Sinne eines dauerhaft eingerichteten Raumes für das hochschuldidaktische Prinzip des Forschenden Studierens. In Workshops und Begleitseminaren können Studierende hier Forschungsmethoden kennenlernen, sich kreativ in der Dateninterpretation erproben und eine Forschungsberatung in Anspruch nehmen. Als Konzept befasst sich die ForwerkEW mit curricularen Systematisierungen von Forschungsbezügen und deren Digitalisierung.

Doch wie kann angesichts einer geforderten Digitalität der Lehre die Werkstattidee mit digitalen Medien unterstützt werden? In diesem Beitrag skizzieren wir drei aktuelle Herausforderungen der Lehrer*innenbildung und zeigen, wie die Forschungswerkstatt darauf mit curricularen Implementierungen und einer Onlineplattform reagiert.

\section{Aktuelle Herausforderungen in der Lehrer*innenausbildung}

Die ForwerkEW wird insbesondere von Studierenden der lehrerbildenden Studiengänge im Fachbereich Bildungs- und Erziehungswissenschaften frequentiert und versucht, mit ihren Angeboten auf drei aktuelle Herausforderungen der reflexiven Lehrer*innenbildung zu reagieren: (a) auf die gebotene Forschungsorientierung universitärer Lehre, (b) auf die bildungspolitische Forderung, Digitalisierungsstrategien für die universitäre Lehre zu entwickeln, sowie (c) auf die Notwendigkeit, angesichts der Heterogenität der Studierenden inklusive Lernformate zu entwickeln.

\subsection{Die gebotene Forschungsorientierung universitärer Lehre}

Das Lehramtsstudium als universitäre Phase der Lehrer*innenbildung hat einen doppelten Anspruch: den der Berufsvorbereitung und den der Wissenschaftlichkeit. Ein zentrales Element dieser ersten Professionalisierungsphase ist der Aufbau einer forschenden Haltung, um sich möglichst unvoreingenommen und mit einer offenen, fragenden Herangehensweise jenseits einer unmittelbaren Verwertungslogik in der Praxis einzuüben und professionsbezogenes Wissen zu erlangen. Die gebotene Forschungsorientierung der Lehre wird mit dem hochschuldidaktischen Konzept des Forschenden Lernens umgesetzt, das seit der Jahrtausendwende erneut eine breite Resonanz erfährt und an den Hochschulen in zahlreichen Kon- 
zepten umgesetzt wird (im Überblick Huber \& Reinmann, 2019; Hoffmeister et al., 2020; Mieg \& Lehmann, 2017; Neuber et al., 2018). Der Begriff des Forschenden Lernens enthält - obwohl im Diskurs häufig verwendet - eine konzeptionelle Unschärfe und Beliebigkeit. Er wird für viele Variationen studentischer Forschungsbezüge verwendet (Huber, 2014). Mit dem Begriff des Forschenden Studierens unternehmen wir in der bremischen ForwerkEW im breit gefächerten Diskurs zum Forschenden Lernen eine wissenschaftliche sowie professionsbezogene Profilschärfung und verstehen darunter ein

hochschuldidaktisches Prinzip der universitären Ausbildungsphase der Lehrer*innenbildung, durch das Studierende mittels eigener (und begleiteter) Forschungsaktivitäten eine für ihren zukünftigen schulpädagogischen Beruf relevante forschende Haltung, Reflexivität und Professionswissen erlangen können. (Thünemann et al., 2020, S. 124).

Forschungswerkstätten sind traditionell Orte des Forschenden Lernens. Als Antwort auf die bildungspolitisch geforderte Forschungsorientierung universitärer Lehre realisiert die bremische ForwerkEW derzeit das Vorhaben, Forschendes Studieren zu einem umfassenden Lehrprofil eines Studienganges zu entwickeln. Das Projekt GOresearch fokussiert den erziehungswissenschaftlichen Anteil des lehramtsbezogenen Studienganges für Gymnasium/Oberschule und konzipiert eine curriculare Einbettung des Forschenden Studierens. Dabei gilt es, Forschendes Studieren nicht nur als Additivum auf der Ebene einzelner Seminare aufzuführen, sondern systematisch in den Modulablauf curricular zu implementieren. Dazu bedurfte es einer Zusammenarbeit aller Verantwortlichen über Modulgrenzen hinweg. Ausgehend vom „Zürcher Framework“ (Tremp \& Hildbrand, 2012), einem Rahmenkonzept für die systematische Verknüpfung von Forschung und Lehre, konzipierten wir zunächst ein Etappenmodell des Forschenden Studierens. Dieses Modell zeigt charakteristisch das Verständnis der ForwerkEW zum Forschenden Studieren, da es neben den gängigen Forschungsetappen (selbst-) reflexive Momente des Forschens, wie beispielsweise die Arbeit mit den eigenen Präkonzepten, expliziert (siehe Abb.1). 


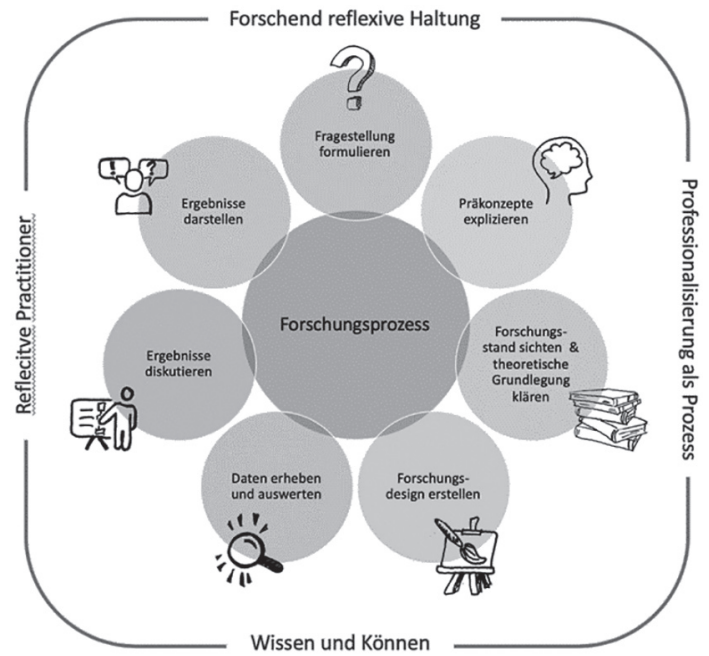

Abb. 1: Etappenmodell der bremischen Forschungswerkstatt Erziehungswissenschaft zum Forschenden Studieren

In einem zweiten Schritt wurde dieses Etappenmodell über die vorhandene Modulstruktur des betreffenden Studienganges gelegt, sodass in jedem Modul einzelne Etappen des Forschungsprozesses verstärkt thematisiert werden. Zur inhaltlichen Profilierung der Module wurden alle Module dem schematischen Vierschritt Annäherung - Überblick - Vertiefung - Anwendung zugeordnet (siehe Abb. 2).
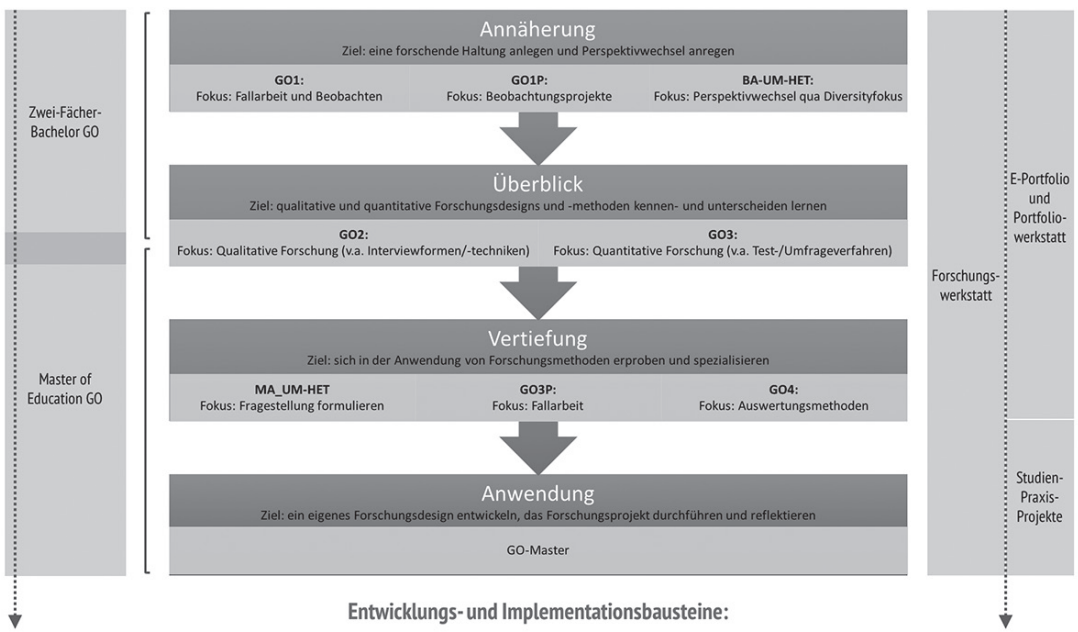

Abb. 2: Zusammenführung des Etappenmodells mit der Modulstruktur des Studiengangs 
Curriculare Implementierungen leben von der Verständigung aller Beteiligten über Modulgrenzen hinweg. Damit die neue Systematik mit Leben gefüllt werden kann, wurden in einem letzten Schritt Materialien für die einzelnen Module, aber auch ein Leadsheet für die Modulbeauftragten zum gemeinsamen Verständnis des Forschenden Studierens erarbeitet. Auf diese Weise können, so unser Ansinnen im GOresearch, Studierende im Durchgang aller Module eine Zusammenhangsperspektive auf das Forschende Studieren entwickeln, die sie spätestens für ihre empirische Masterarbeit benötigen. Dieses curriculare Programm befindet sich derzeit in der ersten Anwendungsphase und wird mit Schulungen und Support für die Modulkoordinator*innen begleitet.

\subsection{Der Ruf nach einer „digitalen Souveränität“ des Einzelnen}

Digitalisierung wird in (hoch-)schulischen Bildungszusammenhängen immer bedeutsamer, sowohl in eigenen Lehr-Lernprozessen als auch in bildungspolitischen Strategien. Seit einigen Jahren nimmt im Diskurs zur Digitalisierung der Begriff der „digitalen Souveränität" eine zentrale Position ein (Friedrichsen et al., 2016; Pohle, 2020; VbW, 2018). Dieser Begriff umfasst viele Dimensionen (staatliche, wirtschaftliche und individuelle) und steht als Kurzformel für die

Fähigkeit von Individuen sowie von staatlichen oder wirtschaftlichen Institutionen, selbstbestimmt digitale Technologien zu nutzen und ihre jeweiligen Rollen in Zeiten der Digitalisierung selbständig und sicher auszuüben zu können (Pohle, 2020, S. 3).

Neben den notwendigen digitalen Kompetenzen gehören zur digitalen Souveränität auch ein Verständnis von potentiellen Gefahren der Digitalisierung, das Wissen und Berücksichtigen notwendiger Sicherheitsaspekte sowie die Reflexion über die Darstellung der eigenen Person in der digitalen Welt (VbW, 2018). Ähnlich formuliert die KMK dieses in ihrem Strategiepapier „Bildung in der digitalen Welt" mit den sechs Kompetenzbereichen Suchen, Verarbeiten und Aufbewahren, Kommunizieren und Kooperieren, Produzieren und Präsentieren, Schützen und sicher Agieren, Problemlösen und Handeln sowie Analysieren und Reflektieren (KMK, 2017). Das bayrische Expert*innengremium „Aktionsrat Bildung" geht in seinem Gutachten zur Digitalen Souveränität und Bildung der Frage nach, welche Beiträge die einzelnen Bildungsphasen für die Entwicklung der digitalen Souveränität des Einzelnen leisten können (VbW, 2018). Für die universitäre Lehrer*innenbildung entsteht mit dieser Frage ein Prozess, der darin mündet, dass angehende Lehrkräfte an der Universität eine digitale Souveränität ausbilden, die sie dazu befähigt, später im Beruf Lernende auf ihrem Weg zu einer digital souveränen Handlungsfähigkeit zu unterstützen. Hochschullehrende müssen sich demnach die Frage stellen, wie sie Lehramtsstudierende für diese zukünftigen Aufgaben vorbereiten können - und welchen Support sie ihrerseits 
für die Hochschullehre benötigen. Dazu gehört es, die Bedingungen, die sich aus einer heterogenen Dozierenden- und Studierendenschaft ergeben, angemessen zu berücksichtigen.

\subsection{Die Entwicklung inklusiver Lernformate unter Berücksichtigung der Heterogenität von Studierenden}

Es sind die unterschiedlichen Lebenszusammenhänge, Bildungsbiographien und Zugangsvoraussetzungen bei zugleich höheren Studierendenzahlen, welche die gegenwärtige Heterogenität der Studierenden mehr noch als vor einigen Jahren prägen und eine Herausforderung in der Lehre darstellen. Nach dem derzeitigen Verständnis von Heterogenität wird diese nicht mehr als zu bearbeitendes Defizit, sondern als Ressource begriffen. Das hat weitreichende Konsequenzen für die Lehre: Erforderlich ist ein Abschied von der Vorstellung, die heterogenen Studierenden insbesondere in der Studieneingangsphase an homogene Studienstrukturen anzupassen, ohne im Studium Angebote und curriculare Vorgaben adaptieren zu müssen (Hanft, 2015). Gefordert sind individuelle Lehr-Lernkonzepte, die auf die Heterogenität Studierender gezielt antworten, barrierefreie Zugänge in der Lehre ermöglichen und dies ohne die Zielsetzung universitärer Lehre, also Professionswissen zu erlangen und eine forschende Haltung aufzubauen, zu vernachlässigen. Auch auf diese Herausforderung scheint Forschendes Studieren eine Antwort zu haben: Satilmis zufolge berücksichtigt Forschendes Studieren Heterogenität insofern, als „es Gestaltungsräume bietet und verschiedene Lernund Kompetenzentwicklungsprozesse anspricht, die an den Befähigungen und Bedarfen der Studierenden anknüpfen“ (Satilmis, 2017, S. 422). Dies ermöglicht Forschendes Studieren durch die relativ selbstgesteuerten Arbeitszyklen des Forschens. Zusätzlich stelle Forschendes Studieren durch die im Konzept inhärente Multiperspektivität und Methodenvielfalt Heterogenität als Konzept selbst stärker in den Vordergrund (ebenda, S. 425).

Aus der gegenseitigen Bezugnahme der Konzepte „Forschendes Studieren“ und „Digitale Medien“ können kreative Lehr-Lernarrangements entstehen, die aber auch zu Überforderungsstrukturen führen können - sowohl bei den Studierenden als auch den Lehrenden (Reinmann et al., 2019). In der Konzeption solcher hochschuldidaktischer Formate muss berücksichtigt werden, dass bei der Umsetzung des Forschenden Studierens mit digitalen Medien zwei voraussetzungsreiche Konzepte (Forschendes Studieren und Digitale Medienbildung) verwoben werden, wobei eine etwaige Überforderung in der Gleichzeitigkeit unterschiedlicher Lernformen liegt (Hofhues, 2017). Es ist bei der Gestaltung und Nutzung von digital unterstützter Lehre daher zentral, diese Angebote möglichst inklusiv zu gestalten, zunächst bei den digitalen Kompetenzen der Lehrenden anzusetzen und verlässliche Support-Strukturen für die Lehrenden aufzubauen (Vereinigung der Bayerischen Wirtschaft, 2018, S. 27). 
Ein Versuch, die Herausforderungen des Forschenden Studierens, der digitalen Medienbildung und der Entwicklung inklusiver Lehr-Lernformate zusammenzubringen, stellt das digitale Online-Studienangebot „BOOC - Blended Open Online Courses“ zum Forschenden Studieren dar, das im Folgenden in seinen zentralen Aspekten vorgestellt wird.

\section{Forschendes Studieren mit BOOC}

Das an der Universität Bremen entstandene Portal „BOOC - Blended Open Online Courses" ist ein interaktives digitales Studienangebot zum gesamten Zyklus des Forschenden Studierens mit didaktisch aufbereiteten Materialien, die auf unterschiedlichen Anforderungsniveaus an Forschung heranführen. BOOC bietet durch die Bereitstellung von wissenschaftlichen Texten, Erklärvideos, Interviews u. v. m. einen Überblick über den gesamten Forschungszyklus und stellt für alle Phasen des Forschungsprozesses Inhalte und Tools bereit. Von der Entwicklung einer Fragestellung bis hin zur Erstellung der M. Ed.-Abschlussarbeit finden sich auf der Plattform Angebote für individuelle Selbstlernphasen bis hin zu kollaborativen Arrangements, wie etwa der Analyse von Datenmaterial im Team. Neben dem „klassischen“ medialen Angebot wie Texten oder Videos stellen interaktive Tools (E-Science-Tools) ein Kernstück von BOOC dar, mit denen sich das neu erworbene Wissen direkt in Handlungen umsetzen lässt. Die Inhalte und Tools zum Forschenden Studieren sind innerhalb von drei aufeinander bezogenen und miteinander verknüpften Ebenen organisiert: Die Vermittlung, die Partizipation und die Präsentation:
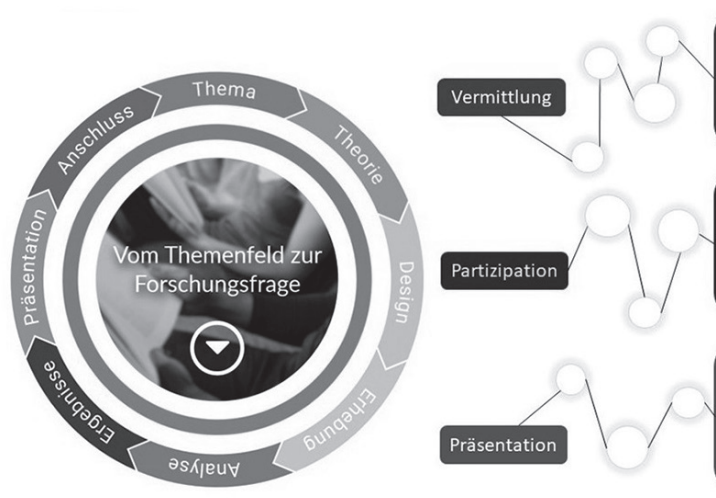

Texte, Linklisten, Erklärfilme

(Tutorials), interaktive Videos und

Grafiken zu Forschungsmethoden und

-designs (qualitativu. quantitativ)

Individuelle und kollaborative

E-Science-Tools (Elektronische

sWerkzeugkästenı z. B. zur Text-und

Bild-analyse)zur Ideenfindung,

Erhebung und Analyse von

Datenmaterial, u.a.

Plattform zum Teilen von Prozessdokumenten und Forschungsergebnissen.

Fakultativ wählbarer Sichtbarkeitsstatus über Seminarebene hinaus.

Abb. 3: Drei Ebenen der Inhalte und Tools in BOOC 
So können Studierende in BOOC beispielsweise zunächst Wissen zum Leitfadeninterview erlangen, im nächsten Schritt eigene Interviewfragen sammeln, diese kriteriengeleitet überprüfen, per Drag \& Drop in eine thematische Reihenfolge bringen und schließlich für den Austausch im Seminar über den PDF-Export speichern. Mit einem weiteren Tool lässt sich dann der tatsächliche Leitfaden strukturieren und erstellen, wie er in der Interviewsituation zur Anwendung kommen kann.

Durch die Arbeit mit BOOC erweitern Lehramtsstudierende zudem ein Handlungsspektrum, das für eine „Bildung in der digitalen Welt“ (Kultusministerkonferenz KMK, 2016) im Kontext des schulischen Berufsfeldes relevant ist (z. B. Prozesse des ELearning, Blended-Learning, digital-kooperative Lernformen). Das digitale Angebot von BOOC hat somit nicht nur zum Ziel, die Prozesse des Forschenden Studierens zu unterstützen, sondern adressiert in seinem Anwendungsbezug auch die Entwicklung und Herausforderung digitaler Kompetenzen. Die Methoden der Aneignung werden in diesem Prozess also gleichsam zu seinem Inhalt (Reinmann, 2011).

Anhand des Angebots zum Forschungsstil der reflexiven Grounded Theory (rGTM) wird im Folgenden vorgestellt, wie in BOOC Forschungshandlungen im Rahmen Forschenden Studierens auf unterschiedlichen Anforderungsniveaus initiiert und damit für eine heterogene Studierendenschaft adaptiv nutzbar gemacht werden.

Beispiel: Digitalisierte und inklusive Lehr-Lernfomate zum Forschenden Studieren mit der reflexiven Grounded Theory

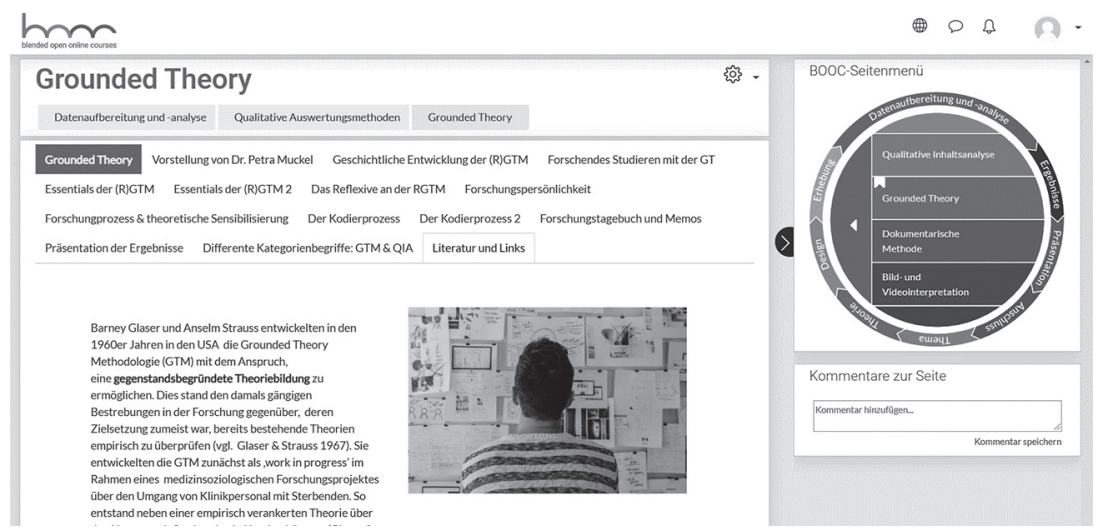

Abb. 4: Digitalisierte und inklusive Lehr-Lernformate zum Forschenden Studieren mit der reflexiven Grounded Theory 
Der Forschungsstil der rGTM bietet sich wegen seiner Forschungsstrategien zur Bearbeitung der subjektiven Involviertheit von Forschenden im Erkenntnisprozess sehr gut für die reflexiv ausgerichtete Lehrer*innenbildung der Universität Bremen an und ist in BOOC prominent vertreten. Im digitalen Kurs zur rGTM erhalten Studierende und Lehrende anhand kurzer Textbausteine sowie über vertiefende Erklär-Videointerviews mit Dr. Petra Muckel (Mitbegründerin der rGTM; Breuer et al., 2018) eine Einführung in Methodologie und Methode dieses Forschungsstils.

Die Inhalte der Vermittlung und die interaktiven E-Science-Tools sind dabei in didaktischen Lehr-Lernszenarien miteinander verschränkt und stellen sich in BOOC durch eine Aufbereitung in Abschnitten dar, durch die Nutzer*innen selbstständig oder zur Unterstützung der Präsenzlehre navigieren können. Die vielfältigen interaktiven Tools bilden die partizipative Ebene von BOOC ab und erlauben es, in vorstrukturierter Weise forschungsmethodische Handlungsschritte erst am Beispiel nachzuvollziehen, dann selbst zu erproben und einzuüben sowie schließlich auf etwaiges eigenes Datenmaterial anzuwenden. Dabei ist es Nutzer*innen möglich, entsprechend der individuellen Voraussetzungen auf einer selbstgewählten Stufe einzusteigen. Dies ermöglicht es, auch im Rahmen des Forschungsprozesses und der Abschlussphase in einer hybrid gestalteten bzw. digital gestützten Lehre auf die Heterogenität der Studierenden zu reagieren.

Im Methodenkurs zur reflexiven Grounded Theory wird zunächst auf einem ersten Anforderungsniveau mittels einer animierten Interviewpassage die Strategie des offenen Kodierens visuell und auditiv vorgestellt. Wie in der Methode üblich, erfolgt nach diesem sichtbar gemachten Prozess des offenen Kodierens der Bezug zu einem Memo als Medium der Reflexion, das sich auf den prominent markierten Code der Sequenz bezieht und in BOOC neben der Interviewpassage eingeblendet wird.

Die Bezüge zwischen der Interviewpassage, den Codes und dem verfassten Memo lassen sich schließlich durch interaktive Verbindungslinien sichtbar machen und werden so für Nutzer*innen nachvollziehbar (siehe Abb. 5). 


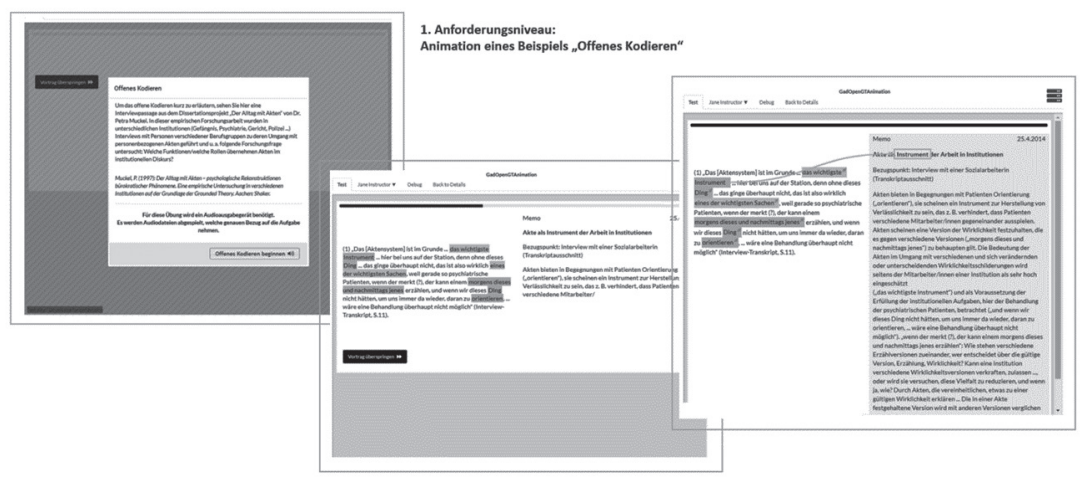

Abb. 5: 1. Anforderungsniveau des E-Science-Tools zum offenen Kodieren in BOOC

Die zweite Stufe des E-Science-Tools zum offenen Kodieren und Memoschreiben mit der rGTM in BOOC bietet die Möglichkeit, an weiterem Beispielmaterial zu bereits markierten Textpassagen eigene Codes zu entwickeln und zu diesen selbst Memos zu verfassen (siehe Abb. 6).

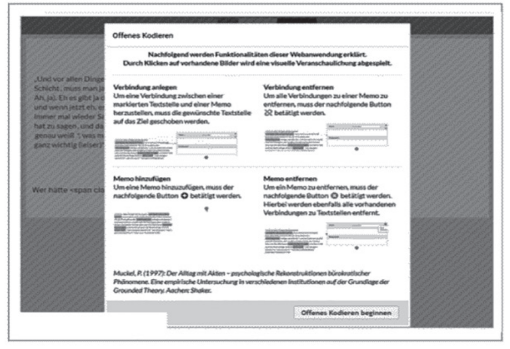

2. Anforderungsniveau:
Codes zu vorausgewähiten Textausschnitten entwickeln, Memos schreiben

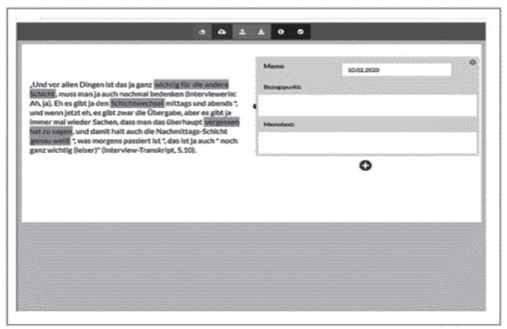

Abb. 6: 2. Anforderungsniveau des E-Science-Tools zum offenen Kodieren in BOOC

Auf der dritten Stufe ist es schließlich möglich, fremdes oder eigenes Datenmaterial selbst einzupflegen und die vorstrukturierte und reduzierte Umgebung des BOOC-Tools zu nutzen, um den zuvor beschriebenen Prozess des offenen Kodierens an einem Datenausschnitt zu erproben (siehe Abb. 7). 


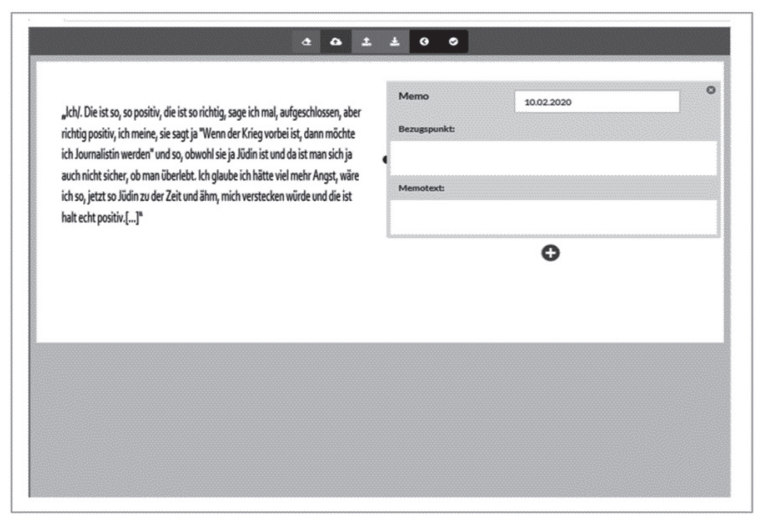

3. Anforderungsniveau: material offen kodieren, Memos schreiben

Abb. 7: 3. Anforderungsniveau des E-Science-Tools zum offenen Kodieren in BOOC

Über einen PDF-Export, den BOOC bereitstellt, lassen sich die eigenen Ergebnisse oder Zwischenergebnisse schließlich speichern und können in einer anschließenden Seminarsitzung präsentiert werden.

\section{Diskussion}

Wir möchten abschließend die Gesamtkomposition der vorgestellten OnlinePlattform BOOC hinsichtlich eröffnender Möglichkeiten und etwaiger Überforderungsstrukturen für die Lehrer*innenbildung diskutieren. Angesichts der in Kapitel 2.2 aufgeführten bildungspolitischen Forderung nach einer digitalen Souveränität der verschiedenen Akteur*innen liegt die Frage nach dem wie der notwendigen Qualifizierung zukünftiger Lehrkräfte auf der Hand. Eine didaktisch aufbereitete Digitalisierung zum Forschenden Studieren ist sicherlich wegen ihrer individuellen Nutzungsmöglichkeiten eine große Chance für die Lehrer*innenbildung. Jedoch liegt eine mögliche Überforderung eben gerade im Zusammenführen von Forschendem Studieren und Digitaler Medienbildung begründet. Diese beiden Konzepte sind per se sowohl für Lehrende als auch für Studierende sehr voraussetzungsreich und mit hohen Ansprüchen verbunden. Nach Hofhues birgt insbesondere die Gleichzeitigkeit dieser ganz unterschiedlichen Lehr-Lehrformen ein hohes Überforderungspotenzial (Hofhues, 2017). Hinsichtlich der Frage eines wie in der Gestaltung von digitalen Medien in inklusiven Lehr-Lernformaten ist daher festzuhalten, dass nicht der alleinige Einsatz digitaler Medien bereits zu einer Verschiebung einer (eher) instruktionslastigen Lehre hin zu einem Lernen mit verstärkter Eigenbeteiligung führt. Gerade gegenwärtig häufig eingesetzte Apps und Tools (wie Cliqr, Kahoot u. ä.), die nach dem 
Frage-Antwort bzw. Quizprinzip funktionieren, eignen sich gut zur Aktivierung in Großveranstaltungen bzw. der Überprüfung von Fachwissen, sind jedoch für Formate des Forschenden Lernens eher nicht förderlich (Hofhues, 2017). Es bedarf also digitaler Lehr-Lernszenarien, die in Forschungszusammenhängen diskursive und iterative Möglichkeiten eröffnen. Auch wird das Lehrangebot nicht durch die bloße Addition eines digitalen Online-Angebots den Flexibilisierungsbedürfnissen einer heterogenen Studierendenschaft gerecht, auch wenn das Angebot einer relativ zeit- und ortsunabhängig nutzbaren Plattform die Präsenzformate dahingehend bereits gewinnbringend erweitern kann, wie erste seminargebundene Evaluationen im Projekt zeigen. Eine Binnendifferenzierung dieses Angebots, die eine Bearbeitung auf verschiedenen inhaltlichen Niveaustufen ebenso zulässt wie die Berücksichtigung unterschiedlich ausgeprägter digitaler Kompetenzen seitens der Studierenden, wird also notwendig.

Rückmeldungen zu dem Angebot von BOOC zeigen, dass nicht für alle Lehrenden und Studierenden Online-Angebote sowohl inhaltlich als auch in der Bedienung an sich selbsterklärend sind - so hoch der Anspruch einer intuitiven Handhabung auch sein mag. Insbesondere interaktive Formate bedürfen also einerseits einer intensiven (face-to-face) Einführung und Betreuung, andererseits aber auch von Seiten der (technischen und mediendidaktischen) Entwicklung umfangreiche personelle Qualifikationen und Ressourcen. So zeigt sich resümierend, dass Online-Plattformen Chancen für innovative Lehr-Lernkonzepte bieten, wegen ihrer Komplexität jedoch Fortbildungen, Beratungen sowie technische Supportstrukturen benötigen, sollen sie umfassend in der Lehre genutzt werden. Diese Rückmeldungen aufgreifend werden derzeit in der ForwerkEW anschlussfähige Kurzworkshops und Tutorials für Lehrende konzipiert, die zunächst fakultätsgebunden angeboten werden sollen.

\section{Literatur}

Breuer, Franz, Petra Muckel u. Barbara Dieris (2018). Reflexive Grounded Theory. Eine Einführung für die Forschungspraxis. 3., vollständig überarbeitete und erweiterte Auflage. Springer.

Doff, S. \& Wulf, M. (Hg.) (2018). Schnittstellen gestalten. Das Zukunftskonzept für die Lehrerbildung an der Universität Bremen. Professionalisierung zum Reflective Practitioner. Resonanz. Magazin für Lehre und Studium an der Universität Bremen.

Forschungswerkstatt Erziehungswissenschaft (2020, 28. August). https://www.uni-bremen.de/fb12/ fachbereich/werkstaetten-des-fb12/forschungswerkstatt-erziehungswissenschaft.html.

Friedrichsen, M., \& Bisa, P.- J. (Hg.) (2016). Digitale Souveränität. Vertrauen in der Netzwerkgesellschaft. Springer VS.

Hanft, A. (2015). Heterogene Studierende - homogene Studienstrukturen. In A. Hanft, O. ZawackiRichter \&W.B. Gierke (Hg.), Herausforderung Heterogenität beim Übergang in die Hochschule (1315). Waxmann.

Hoffmeister, T., Koch, H., \& Tremp, P. (Hg.) (2020). Forschendes Lernen als Studiengangsprofil. Zum Lehrprofil einer Universität. Springer VS. 
Hofhues, S. (2017). Forschendes Lernen mit digitalen Medien. In H.A. Mieg \& J. Lehmann (Hg.), Forschendes Lernen. Wie die Lehre in Universität und Fachhochschule erneuert werden kann (410418). Campus Verlag.

Huber, L. (2014). Forschungsbasiertes, Forschungsorientiertes, Forschendes Lernen: Alles dasselbe? Ein Plädoyer für eine Verständigung über Begriffe und Unterscheidungen im Feld forschungsnahen Lehrens und Lernens. Hochschulforschung 1+2, 22-29.

Huber, L., \& Reinmann, G. (2019). Vom forschungsnahen zum forschenden Lernen an Hochschulen. Wege der Bildung durch Wissenschaft. Springer VS.

Kultusministerkonferenz (2017). Bildung in der digitalen Welt. Strategie der Kultusministerkonferenz. https://www.kmk.org/fileadmin/Dateien/veroeffentlichungen_beschluesse/2018/Strategie_Bildung_in_der_digitalen_Welt_idF__vom_07.12.2017.pdf.

Kulturministerkonferenz (2016). Kompetenzen in der digitalen Welt. https://www.kmk.org/fileadmin/Dateien/pdf/PresseUndAktuelles/2017/KMK_Kompetenzen_in_der_digitalen_Welt_neu_26.07.2017.html.

Landesregierung Nordrhein-Westfalen (2019). Medienkompetenzrahmen NRW. https://medienkompetenzrahmen.nrw/fileadmin/pdf/LVR_ZMB_MKR_Rahmen_A4_2019_06_Final.pdf.

Mieg, H.A., Lehmann, J. (2017). Forschendes Lernen. Wie die Lehre in Universität und Fachhochschule erneuert werden kann. Campus Verlag.

Müller-Naendrup, B. (1997). Lernwerkstätten an Hochschulen. Ein Beitrag zur Reform der Primarstufenlehrerbildung. Peter Lang.

Neuber, N., Paravicini, W., Stein, M. (2018): Forschendes Lernen - the wider view. Eine Tagung des Zentrums für Lehrerbildung der Westfälischen Wilhelms-Universität Münster vom 25. Bis 27. 09. 2017. WTM.

Pohle, J. (2020). Digitale Souveränität. In. T. Klenk, F. Nullmeier \& G. Wewer (Hg.), Handbuch Digitalisierung in Staat und Verwaltung (1-13). Springer VS.

Reinmann, G. (2011). Blended Learning in der Lehrerausbildung. Didaktische Grundlagen am Beispiel der Lehrkompetenzförderung. Seminar BAK, 17 (3), 7-16.

Reinmann, G., Lübcke, E., \& Heudorfer, A. (2019). FiDeS. Die Geschichte unseres Verbundprojektes. In Dies. (Hg.), Forschendes Lernen in der Studieneingangsphase (1-10). Springer VS.

Satilmis, A. (2017). Forschendes Lernen und Heterogenität. In H. A. Mieg \& J. Lehmann (Hg.), Forschendes Lernen. Wie die Lehre in Universität und Fachhochschule erneuert werden kann (419428). Campus Verlag.

Thünemann, S., Schütz, A., \& Dogmus, A. (2020). GOresearch - Konzeptionelle Zugänge zum Forschenden Studieren im erziehungswissenschaftlichen Lehramtsstudium. In T. Hoffmeister, P. Tremp \& H. Koch (Hg.), Forschendes Lernen als Studiengangsprofil - Zum Lehrprofil einer Universität (123-136). Springer VS.

Tremp, P., \& Hildbrand, T. (2012). Forschungsorientiertes Studium - universitäre Lehre: Das „Zürcher Framework“ zur Verknüpfung von Forschung und Lehre. In T. Brinker \& P. Tremp (Hg.), Einführung in die Studiengangentwicklung (Blickpunkt Hochschuldidaktik, Bd. 122, 101-116). Bertelsmann.

Vereinigung der Bayerischen Wirtschaft e.V. (Hg.) (2018). Aktionsrat Bildung: Digitale Souveränität und Bildung. Gutachten. Waxmann. 